\title{
Fluid Mechanics and Fluid Power (FMFP)
}

\section{FOREWORD}

This special issue of Sadhana is a compilation of invited papers from the 42nd National Conference on Fluid Mechanics and Fluid Power (FMFP), held at National Institute of Technology,. Surathkal, Karnataka, during December 14-16, 2015. Broadly speaking, Fluid Mechanics is a branch of continuum mechanics that describes the motion of fluids (liquids and gases). The subject has a long and rather rich history, starting perhaps with Archimedes' "Eureka" moment. Importantly, solution for some of the most challenging problems being faced by our society today, e.g., prediction of climate, harnessing of renewable energy (e.g., via wind, hydrokinetic generators), creating low-cost healthcare (e.g., via point-of-care medical testing) and improvement of energy efficiency of fluid power systems, depends on improving our understanding of Fluid Mechanics.

Fluids are ubiquitous in both nature and technological applications, and their dynamics cover a range of time and length scales. Therefore, Fluid Mechanics is used by researchers in a diverse set of disciplines, in almost all branches of science and engineering. Arguably, the subject has benefited tremendously from the cross-pollination of ideas between research communities in engineering and science. For instance, Large Eddy Simulation (LES) models, now used widely by engineers to efficiently simulate turbulent flows, was in fact first devised by Joseph Smagorinsky, a researcher in atmospheric science. Conversely, Sir James Lighthill, who founded the field of acoustics and aeroacoustics early in his career, later went on to make seminal contributions towards explaining the mechanics of biological swimmers and flyers. Due to this widespread exchange of ideas, research problems in Fluid Mechanics have remained rich and challenging. It is now very common for graduate students within the same research group to work on diverse set of problems, involving topics like fluid-structure interaction, multiphase flow, turbulence, bio-fluid dynamics, atmospheric flows, microfluidic flows, and so on.

Experimental, computational and analytical techniques have formed the backbone of Fluid Mechanics. Over the last few decades, mainly due to the rapid improvement in computational efficiency, cameras, optics and instrumentation, both computational and experimental techniques have improved significantly, allowing researchers in Fluid Mechanics to build better mechanistic and analytical models for processes involving dynamics of fluids.

Authors were invited to this special issue based on the novelty and rigour of their work, presented in talks and papers at the conference. We also invited three eminent researchers in Fluid Mechanics, and their respective research groups, to contribute review papers on selected topics. All papers in this issue were accepted for publication in Sadhana after the standard review process by referees; we thank all the referees of this special issue for their effort and timely reviews.

The manuscripts in this issue reflect the diversity of topics and methodology in modern-day Fluid Mechanics. In a review paper relevant to atmospheric sciences by Prof. Rama Govindrajan's group, Ravichandran et al. have elucidated on mechanisms behind clustering of droplets in atmospheric clouds, an important process in rainfall prediction. In Pai et al., the effect of pulsations on the motion of particles in vortices is explored, with the goal of enhancing mixing. In a paper related to environmental fluid dynamics, Shah et al. measure the thermal dispersion of effluents in a lake, and also perform numerical simulations to further analyse the phenomenon.

Several of the papers have used state-of-the-art computational techniques to study problems related to multi-phase flows and fluid-structure interaction. Nair and Tomar have used a novel formulation of Smooth Particle Hydrodynamics (SPH) to study the challenging problem of entry of solids in water. Patel and Shukla use a phase-field technique to study the breakup and detachment of liquid bridges, seen in processes such as printing and coating of paints with brushes. Namshad et al. use level-set-based methods to study the effect of wavelength on fish locomotion, which leads to better understanding of thrust in fishes as well as robotic swimmers. Sarkar et al. use an improved mass-conserving immersed-boundary method to study unsteady blood flow in arteries, with the aim of simulating flow through stenosis. Eswaran et al. use a sigma-transformation-based fluid-structure-interaction solver to evaluate a multiple-tuned-liquid-damper system for buildings.

The physics of multi-phase flow have been highlighted in the following papers. The review paper by Kirti Chandra Sahu describes the dynamics of rising bubbles in viscosity-stratified flows, a common phenomenon in, for example, food processing. The review paper by Sameer Khandekar's group, authored by Srinivas and Khandekar, examines the dynamics and patterns of two-phase flows in microchannels, which has applications in biomedical engineering and electronic cooling. The paper by Garai et al. experimentally examines the atomization of different types of liquid fuels, with the goal of optimizing the energy efficiency of combustors. 
The following papers demonstrate the potential of combining Computational Fluid Dynamics (CFD) with experiments towards design of fluid power systems. Asim and Mishra perform Large Eddy Simulation (LES) of centrifugal pumps to examine the effect of blade-tongue interactions in the pump on pressure head fluctuations and validate their results with experiments. Sanand et al. use CFD and experiments to optimize centrifugal seals in fluid machinery, so that sealing ability is maximized, while minimizing power consumption.

We hope that papers in this special issue will have significant impact in the field of Fluid Mechanics, and we thank the editors of Sadhana for inviting us to review and bring out this issue.

April 2017

AMITABH BHATTACHARYA

Department of Mechanical Engineering, Indian Institute of Technology Bombay, Mumbai 400076, India

e-mail: bhattach@gmail.com
JYOTIRMAY BANERJEE

Department of Mechanical Engineering, Sardar Vallabhbhai National Institute of Technology,

Surat 395007, India

e-mail: jbaner@med.svnit.ac.in

(Guest Editors) 